\title{
Prolegomena für ein phasenübergreifendes Lehramtsportfolio (nicht nur) für Fremdsprachenlehrende
}

\author{
Eva Burwitz-Melzer (Gießen/Deutschland)
}

\section{Einleitung}

Dieser Artikel beschäftigt sich mit Vorüberlegungen zu einem neuen Lehramtsportfolio, das von allen Lehrämtern, für die Ausbildung in allen Schulfächern und in der Ausbildung aller Studienanteile für das Lehramt genutzt werden kann. Nach einem kurzen Blick auf die sehr disparate Ausgangslage, die momentan in der Lehramtsausbildung in Deutschland herrscht, werden zunächst übergreifende Professionalisierungsziele in der Lehramtsausbildung dargestellt. Es wird dann sukzessive aufgezeigt, wie diese Ziele mit Hilfe eines kompetenzorientierten Lehramtsportfolios in allen Phasen der Lehramtsausbildung umgesetzt werden könnten. ${ }^{1}$

\footnotetext{
${ }^{1}$ Kurze Teile dieses Artikels sind in den Aufsatz Ein phasenübergreifendes Portfolio in der Lehramtsausbildung eingegangen: Burwitz-Melzer, Eva (2018): Ein phasenübergreifendes Portfolio in der Lehramtsausbildung. In: Burwitz-Melzer, Eva; Riemer, Claudia; Schmelter, Lars (Hrsg.): Rolle und Professionalität von Fremdsprachenlehrpersonen. Arbeitspapiere der 38. Frübjabrskonferenz zur Erforschung des Fremdsprachenunterrichts. Tübingen: Narr, 21-31.
} 


\section{Zur Ausgangslage in der ersten und zweiten Phase der Lehramtsausbildung}

Der in der Konferenz von Bologna vereinbarte Prozess zur Angleichung und Modularisierung der Studiengänge in Europa im ersten Jahrzehnt des Jahrtausends hat auch auf das Studium aller Lehrämter einen großen Einfluss gehabt. In den meisten Bundesländern hat man sich für ein Bachelor- und Master-Modell entschieden, in dem erst in der Master-Phase die Weichen endgültig auf das Lehramt gestellt werden und bis zum Bachelorabschluss nur wenige fachdidaktische Anteile in den zwei studierten Schulfächern absolviert werden. In einigen Bundesländern ist man bei einem Staatsexamensabschluss geblieben, der in traditioneller Manier die einzelnen Anteile der Fachwissenschaft und Fachdidaktik, sowie der vier Bildungswissenschaften von Studienbeginn bis zum Ersten Staatsexamen nebeneinander anbietet. Die Studierenden durchlaufen in diesem letzteren Ausbildungsmodell ein sechs-, sieben- oder achtsemestriges Studium, je nach Lehramtsspezifikation. Beiden Modellvarianten gemeinsam sind Praxisphasen, die neben einem Orientierungspraktikum in der Regel ein langes Praxissemester oder zwei Blockpraktika (am Anfang und gegen Ende des Studiums) vorsehen. Abgeschlossen wird die erste Ausbildungsphase durch die Erste Staatsexamensprüfung oder durch einen Master of Education. Der ersten, vorwiegend wissenschaftlich orientierten Phase der Lehramtsausbildung stehen in den einzelnen Bundesländern zweite Ausbildungsphasen im Referendariat gegenüber, die sich durch unterschiedliche Länge (zwischen 18 und 24 Monaten) und eine unterschiedliche Prüfungsbelastung beim Zweiten Staatsexamen auszeichnen. Insgesamt weist somit die deutsche Lehramtsausbildung für alle Lehrämter und Fächer während der ersten beiden Phasen eine uneinheitliche Ausbildungssituation auf. Diese wird durch eine stetig ansteigende Zahl von Quer- und Seiteneinsteigern in der Lehramtsausbildung noch verkompliziert, da diese Gruppe von Auszubildenden mit ausländischen Studienabschlüssen oder nur mit fachwissenschaftlichen Kompetenzen ausgerüstet oft in einem höheren Alter in höhere Semester oder gar erst in die zweite Ausbildungsphase „eingefädelt“ werden, um Planungslücken bei der Lehrerausbildung auszugleichen oder innereuropäische Fluktuation zu ermöglichen. Die Fluktuation zwischen den deutschen Bundesländern wird durch diese komplexe Situation maßgeblich erschwert, da es diffizil ist, die Kompetenzen und die Leistung eines ausgebildeten Lehrers/einer ausgebildeten Lehrerin aus einem anderen Bundesland nach dem Ende der zweiten Ausbildungsphase genau einzuschätzen. 


\section{Das Lehr- und Lernziel des guten Lehrers/der guten Lehrerin: Bildungspolitische Vorgaben zwischen pädagogischen und fachdidaktischen kompetenzorientierten Ansätzen}

Die starke Diversität der deutschen Ausbildungsgänge zum Lehrer/zur Lehrerin in allen schulischen Einsatzbereichen ist vor dem föderalistischen Hintergrund zwar erklärbar, aber nicht unbedingt zielführend. Denn so unterschiedlich die Lehramtsstudiengänge auch strukturiert sind, so werden sie doch insgesamt stets von denselben Fragen und Zielen geprägt:

- Was macht einen guten Lehrer/eine gute Lehrerin für die verschiedenen Schulformen und Lern-/Lehrbedarfe aus?

- Auf welchem Wege kann man das Ziel eines guten Lehrers/einer guten Lehrerin in der zweiphasigen Lehrerausbildung erreichen, wenn pädagogische, fachwissenschaftliche und fachdidaktische Belange gleichermaßen eine Rolle in der Ausbildung spielen sollen? (vgl. auch Korthagen 2004: 78f.)

- Wie kann das Ziel des guten Lehrers/der guten Lehrerin bestmöglich über die zwei Lernphasen verteilt angestrebt werden, auch wenn die Voraussetzungen der Lernenden sehr unterschiedlich sind?

Dies ist kein ganz einfaches Unterfangen, denn die Forschungen zur Ausbildung von Lehrkräften, insbesondere zu Englischlehrkräften, sind nicht zahlreich, ist es doch schwierig in der ersten und zweiten Phase zu authentischen belastbaren Daten zu gelangen, da hier oft Persönlichkeitsrechte und forschungsethische Aspekte dem Forschungsdesiderat entgegenstehen. Die größte quantitative Querschnitt- und Längsschnitt-Untersuchung zur Ausbildung professioneller Kompetenzen im Studienverlauf hat die Forschergruppe um Sigrid Blömeke vorgelegt, die an acht Hochschulen der Bundesrepublik die professionelle Entwicklung von Lehramtsstudierenden in den Fächern Deutsch, Englisch und Mathematik sowie Pädagogik beforscht hat (vgl. TEDS-LT-Studie Blömeke et al. 2013: 7-24).

Konzeptionell fokussiert sich die Lehramtsausbildung in den letzten hundert Jahren in den meisten Ländern auf einige wenige pädagogische Modelle, das CraftModell, das Applied Science Modell und das Konzept des reflective practitioner, die unterschiedliche Foki auf Handlungswissen und wissenschaftliche Konzepte sowie die Reflexionsfähigkeit von Lehramtsstudierenden legen. Während das Craft-Modell eine Art Meister-Schule beschreibt, in der die Auszubildenden von Experten und Expertinnen lernen, indem sie ihnen zuschauen, verfolgt das Applied-Scrience-Modell einen Ansatz, der auf einer spezifischen Lerntheorie beruht (z.B. dem Behaviorismus) und diesen einüben lässt. In den 1980er- und 1990er-Jahren und noch darüber hinaus wurde besonders das reflective practitioner-Modell favorisiert, das nach Schoen (1983) ein Zusammenspiel von Wissen über das Lehren und Lernen, 
Lehrerfahrung und Reflexion propagiert (vgl. Burwitz-Melzer 2004, Grenfell 2012: 168). Dieses Modell hat die Lehramtsausbildung, auch die Ausbildung von Sprachenlehrenden maßgeblich beeinflusst, denn es gibt heute wohl kaum einen Lehramtsstudiengang, der das Element der Reflexion nicht aufgreift und an einer zentralen Stelle im Ausbildungsgang verortet. Aktuelle Konzepte beziehen sich meist auf ein kompetenzorientiertes Modell (CBTE, competence based-teacher-education), das eine Liste von Kompetenzen als Maßstab für gutes Lehrerhandeln vorgibt und diese Kompetenzen im Laufe der Ausbildung fördern möchte.

It is, of course, unlikely that any of these ever exists in a pure form, but it is important to recognise that they do each lead to clear differences of principles, processes and practice. Each one can also be interpreted and used in different ways. Competence-basedteacher-education, for example, can be a top-down set of prescribed content items, or a bottom-up constituted list. Similarly, such lists might be used for either self-evaluation or inspection (Grenfell 2012: 168).

Zunächst einmal muss geklärt werden, was man konzeptionell unter dem Begriff der professionellen Kompetenz im Zusammenhang der Lehramtsausbildung versteht. Wie Blömeke unter Rückgriff auf eine Definition von Bromme ausführt, geht er weit über „,deklaratives Faktenwissen“ hinaus (vgl. Blömeke 2013: 11) und „umfasst die einmal bewusst gelernten Fakten, Theorien und Regeln sowie die Erfahrungen und Einstellungen der Lehrerin. [...] Der Begriff umfasst also auch Wertvorstellungen, nicht nur deskriptives und erklärendes Wissen“ (Bromme 1929: 9f., zitiert nach Blömeke 2013: 11). Insofern ist dieses komplexe Konstrukt der professionellen Lehrerkompetenz durch seine Mehrperspektivität auf kognitive und affektiv-motivationale Fähigkeiten durchaus vereinbar mit dem Weinertschen

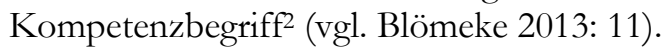

Lehramtsstudierende müssen also durch Reflexion eine Brücke schlagen zwischen kognitiven Wissenselementen und ihren Erfahrungswerten und Einstellungen, die sie bereits zum Studium mitbringen und dort weiterentwickeln. In der Regel gehen heutige Lehramtsausbildende davon aus, dass diese Brücke in Anlehnung an das Modell des reflective practitioner durch Reflexion, also Bewusstmachung, gebaut werden kann (vgl. auch Burwitz-Melzer 2004). Bisherige bildungspolitische Dokumente aus den letzten fünfzehn Jahren, die sich mit der Lehrerausbildung befassen, gehen ebenfalls von einem Kompetenzraster aus, das aber bisher nicht erschöpfend durchdacht erscheint, da sie die unterschiedlichen Aspekte der Fachwissenschaften und -didaktiken sowie der Pädagogik nicht zusammenführen: Zwar

\footnotetext{
${ }^{2}$ Franz E. Weinert definiert Kompetenzen als „die bei Individuen verfügbaren oder durch sie erlernbaren kognitiven Fähigkeiten und Fertigkeiten, um bestimmte Probleme zu lösen, sowie die damit verbundenen motivationalen, volitionalen [die willentliche Steuerung von Handlungen und Handlungsabsichten] und sozialen Bereitschaften und Fähigkeiten, um die Problemlösungen in variablen Situationen erfolgreich und verantwortungsvoll nutzen zu können“" (Weinert 2001: 27f.).
} 
existieren in Deutschland die Standards für die Lehrerausbildung: Bildungswissenschaften (KMK 2004), die ein grobes Raster von Standards für die Kompetenzbereiche Unterrichten, Erziehen, Beurteilen und Innovieren anbieten, doch wurde bei diesem Entwurf nicht bedacht, dass drei der Bereiche, nämlich Unterrichten, Beurteilen und Innovieren nur sinnvoll dargestellt werden können, wenn sie auch stets in fachdidaktische Überlegungen integriert sind, da der pädagogische Ansatz allein für die angehenden Lehrkräfte zu kurz greift. Abgesehen von sehr basalen Fertigkeiten und Wissensbereichen fehlt hier der bereits durch den Filter der Fachdidaktik spezifisch ausgerichtete Blick auf die Materie, die es den angehenden Lehrkräften ermöglicht, die Kompetenzbereiche leichter mit ihren Fächern zu verknüpfen. Auch hat man es in dem Dokument vermieden, eine Stufung von Kompetenzen darzustellen, die unterschiedliche aufeinander aufbauende Niveaus, z.B. ein Anfängerniveau (Beginn bis Mitte des Studiums), ein mittleres Niveau (Beginn des Referendariats) und ein Fortgeschrittenenniveau (Lehrer/Lehrerin im Beruf) umfassen.

Im zweiten großen bildungspolitischen Dokument zur Lehrerbildung, den Ländergemeinsamen inhaltlichen Anforderungen für die Facbwissenschaften und Fachdidaktiken in der Lebrerbildung (KMK 2008) werden die Standards nur in sehr grober Form auf einem Niveau für die Lehrämter der Sekundarstufen I und II angeboten. Eine genauere Beschreibung von Kompetenzprofilen der berufsbildenden Gymnasien und der Sonderpädagogik erfolgt nicht, obwohl sich das Dokument explizit auch auf diese Lehrämter beziehen soll. Die ausgewählten Kompetenzbereiche sind zwar insgesamt ausreichend, da die Sprachpraxis, die Sprachwissenschaft, die Literatur- und die Kulturwissenschaft ebenso wie die Fachdidaktik einbezogen werden. Eine Darstellung des Kompetenzprofils von Sprachenlehrkräften im Grundschullehramt wird aber ausgespart, was zu einem unnatürlichen und wenig überzeugenden Bruch zwischen den verschiedenen Lehrämtern führt. Dass 2014 kurze Anmerkungen für einen heterogenen und inklusiven Unterricht angefügt wurden, konnte weder Lehrkräfte noch ihre Ausbilder/Ausbilderinnen überzeugen und erfüllt eher eine Feigenblatt-Funktion.

Auch das im Jahr 2015 von der KMK herausgegebene Dokument zur Lehrerbildung für eine Schule der Vielfalt: Gemeinsame Empfeblung (KMK 2015) konnte diesen Mangel nicht ausgleichen, denn hier finden sich nur sehr allgemeine programmatische Bemerkungen, die weder eine pädagogische, noch eine fachdidaktische Konzeption darstellen. Eine Hilfestellung für die Ausbildung der Lehrkräfte für eine inklusive Schule, eine Überarbeitung der Fachkompetenzen und pädagogischen Kompetenzen fehlt. 


\section{Kompetenzorientierung und Reflexion: Portfolios in der Lehramtsausbildung}

Bildungsgänge kompetenzorientiert und gleichzeitig auch durch Reflexion der Auszubildenden zu begleiten, ist ein Ansatz, der in der Hochschuldidaktik der USA und Kanada schon seit Längerem mit Portfolios verfolgt wird, ${ }^{3}$ während man sich in Europa erst in den letzten zwei Jahrzehnten intensiv mit dem Gedanken dieses studienbegleitenden Instruments auseinandergesetzt hat. Felix Winter führt in seinem Überblicksartikel „Das Portfolio in der Hochschulbildung“ (2013) fünf verschiedene Portfoliodokumente an, die studienbegleitend in Seminaren oder ganzen Ausbildungsgängen Studienleistungen sammeln, Kompetenzzuwächse verzeichnen und zur Reflexion von Studieninhalten anregen (vgl. Winter 2013: 1618). ${ }^{4}$

Speziell in den Lehramtsstudiengängen gibt es in den deutschen Bundesländern zahlreiche unterschiedliche Portfoliotypen, die veranstaltungs- bis studienbegleitend mit sehr heterogenen Konzepten angelegt sind. Gelegentlich wird solch ein vielversprechender Ansatz dann auch von der Bildungspolitik aufgegriffen: Das Lehrerausbildungsgesetz des Landes NRW macht in der Lehramtsausbildung ein Portfolio in der ersten und zweiten Phase der Ausbildung in Hinblick auf alle Praxisphasen (Praktika, Praxissemester und das Referendariat) verbindlich und verlangt von den Studierenden sowie Referendaren und Referendarinnen fünf unterschiedliche, allerdings inhaltlich miteinander verbundene Portfolios (vgl. Koch-Priewe 2013: 42). Dieser Versuch, ein phasenübergreifendes Portfoliomodell „von oben“ in die Lehramtsausbildung einzubringen, ist allerdings bisher ein Einzelfall. Wieder stoßen wir auf einen länderspezifisch stark ausdifferenzierten Hintergrund, der es erschwert, einen Überblick über europäische Ansätze, Rahmenvorgaben und Portfolio-Prototypen sowie eigens für die deutsche Ausbildungssituation geschaffene und speziell für Sprachenlehrende konzipierte Portfo-

\footnotetext{
3 Als professionelle Dokumentensammlungen für Lehrkräfte haben Portfolios in den USA und Kanada seit vielen Jahren einen festen Stellenwert, indem sie Studienleistungen der angehenden Lehrer/Lehrerinnen und die später erworbenen Qualifikationen sowohl für Bewerbungen als auch zur eigenen Reflexion der Lehrkräfte im späteren Berufsleben dokumentieren (vgl. Campbell et al. 2001, Lyons 1998, Rebel \& Wilson 2002). In diesem Zusammenhang kann ein professionelles Portfolio folgendermaßen beschrieben werden: „Ein Portfolio ist ein zum Leben erwachtes Resumé [wobei im US- und kanadischen Englisch Resumé auch Curriculum Vitae bedeutet; Anm. d. A.]; eine gut durchorganisierte zielorientierte Dokumentation der professionellen Entwicklung eines künftigen Lehrers/einer künftigen Lehrerin sowie deren jeweils erreichte Kompetenz im Unterrichten. Das Portfolio ist somit ein mit Händen greifbarer Nachweis der Kenntnisse, Fertigkeiten und Einstellungen, über die Lehrer als sich entwickelnde Professionelle verfügen“ (Wilson 2001, zitiert nach Rebel \& Wilson 2002: 65).

${ }^{4}$ Er zählt folgende Typen auf, die sich allerdings teilweise überlappen: 1. Seminar- oder Veranstaltungsportfolios, 2. ausbildungsbegleitende oder Studienportfolios, 3. Prüfungsportfolios, 4. Bewerbungs- oder Zulassungsportfolios und 5. Lehrportfolios von Dozenten (vgl. Rebel \& Wilson 2002: 16-18).
} 
lios zu gewinnen. Unbestreitbar ist aber das Portfolio in der Lehramtsausbildung der Universität und darüber hinaus in der zweiten und dritten Phase ein starkes Desiderat, das eine durch stringente Argumente gestützte Befürwortung genießt. Nicht immer allerdings ist es leicht, alle Stakeholder in der Ausbildung von den Vorteilen eines Portfolios zu überzeugen (vgl. für Hessen Neß 2013), da es die vermeintlichen Freiheiten der Hochschullehre etwas einschränkt, indem es die Lehramtsausbildung stärker vereinheitlicht und darüber hinaus auch ein zeitaufwändiges Ausbildungsinstrument ist.

Dabei kommt aus dem europäischen Ausland eine starke Schützenhilfe für das Lehramtsportfolio - gerade für die Fremdsprachenlehrerausbildung. Nach dem Common European Framework of Reference (Council of Europe 2001) und dem European Language Porffolio (ESP; Council of Europe 1997) unterstützte der Europarat die Entwicklung weiterer Instrumente zur Stärkung des Sprachenlernens und -lehrens. Diese Impulse waren geleitet von wirtschaftlichen und kulturellen Motiven, die eine größere Mobilität von Arbeitskräften innerhalb des Binnenmarktes der EU voraussahen und maßgeblich fördern wollten:

The Common European Framework of Reference showed that it was possible to produce a single focal point for guiding the teaching and learning of second languages. Multilingualism and plurilingualism became the new watchwords in European language policy. Key principles in these developments were coherence, flexibility, cohesion and comprehensiveness. It was essential to produce a policy framework that was of genuine specific help in guiding language teaching and learning across Europe, and therefore in many distinct social and cultural contexts. [...] As the European Union continued to deepen and widen, and physical and fiscal barriers were slowly broken down by increased integration, it was becoming apparent that linguistic barriers needed to be tackled more urgently. If Europe's ambition of free mobility for citizens across the union was to be achieved, it was evident that language teaching and learning needed to form part of this project. At the same time, European unity depended on respect for cultural diversity, of which language, as a cultural marker, plays a huge part (Grenfell 2012: 156).

So wie der Common European Framework of Reference eine Grundstruktur für das Lehren, Lernen von Sprachen und das Evaluieren von Lernleistungen auf diesem Gebiet liefert, wurde daneben ein Dokument erstellt, das vergleichbare Rahmenstrukturen für die Ausbildung der Sprachenlehrer und -lehrerinnen liefern sollte: Das von Kelly und Grenfell erstellte European Profile for Language Teacher Education (EPLTE; Kelly \& Grenfell 2004), das aus mehreren europäischen Forschungsprojekten hervorgegangen ist (vgl. Grenfell 2012: 156f.). Es möchte ein Expertendokument sein, das zentrale Schlüsselelemente für eine erfolgreiche Ausbildung 
von Fremdsprachenlehrkräften in unterschiedlichen Kontexten und europäischen Ländern anbieten kann. Die Motivation, die zu diesem Dokument führte, beschreibt Michael Grenfell so:

How might such a language teacher be educated? What would be the values, knowledge, skills and competences that they would share? What would be their field of operations? The Profile project itself set out to answer these questions. [...] The general aim was to develop and provide a formal set of key elements to guide language teacher education in Europe. Such elements would be central to the formation of "The European Language Teacher" referred to above. However, it was recognized that such an individual would often develop as part of in-service training and extensive professional experience. The Profile itself was then to be of use at this expert level, as well as in initial, pre-service courses. The founding principles were neither prescriptive nor proscriptive. "Rigorous flexibility" became our watchword, that is something that was robust enough to be of specific help, but flexible enough to be adaptable (Grenfell 2012: 157).

Richtet sich das hier beschriebene Profile an Ausbilder/Ausbilderinnen und Ausbildungsinstitutionen für europäische Fremdsprachenlehrkräfte, so stellt das von David Newby in Graz etwas später erstellte European Portfolio for student teachers of languages: A reflection tool for language teacher education (Newby et al. 2010) eine Handreichung für die Studierenden des Fremdsprachenlehrberufs dar. Beide Dokumente verbinden die vorberufliche Ausbildung und die Ausbildungskomponenten miteinander, die während der Berufsausübung abgeleistet werden. Beide sind als unmittelbar miteinander verbunden zu betrachten, indem sie sehr ähnliche Kompetenzbereiche abbilden, aber jeweils das betonen, was für den jeweiligen Kontext fokussiert werden sollte (vgl. Newby et al. 2010, Kelly \& Grenfell 2004). Das EPLTE hat dabei einen eher präskriptiven, das EPOSTL einen deskriptiven bzw. reflexiven Charakter.

Das EPOSTL möchte also für die fachspezifische Ausbildung der Sprachenlehrenden einen validen Rahmen und einen Reflexionshintergrund anbieten. Untersucht man das EPOSTL daraufhin, erkennt man, dass hier der Weg der Selbstbeurteilung gewählt wurde, der durch eine umfassende Reflexion des eigenen Wissens und der eigenen Kompetenzen ergänzt wird, um die Professionalisierung der Studierenden voranzutreiben. Die Kategorien der aus vielen FrameworkDokumenten bekannten Kann-Beschreibungen beziehen sich auf sieben Kompetenzfelder, die jeweils noch weitere Unterstrukturen aufweisen: 
- den Lehr-Lernkontext,

- die Methodik des Lehrens,

- die Ressourcen, die zum Lehren und Lernen benötigt werden,

- die Beurteilung des Lernens,

- das selbstständige Lernen,

- die Planung von Unterricht und

- $\quad$ seine Durchführung (vgl. Kelly \& Grenfell 2004).

Eingeführt wird in das EPOSTL durch eine kurze Selbstbeschreibung, die Erwartungen an den Lehrberuf und das Sprachenlehren vorstellt und eine erste Reflexion über Lehr-/Lernzusammenhänge anbietet. In jedem Kompetenzbereich werden in gewissen Zeitabständen dreimal Selbstevaluationen durchgeführt. Ein Dossier hilft den Nachweis über eigene Lernfortschritte zu führen, indem Beispiele aus der Unterrichtsarbeit gesammelt werden können. Damit wird das Dossier auch zu einer Art Dokumentationsplattform. Die Kann-Beschreibungen und die gesammelten Dokumente sollen von den angehenden Lehrern/Lehrerinnen als Reflexionsanlässe genutzt werden. Insgesamt ist das EPOSTL als Instrument des/der Auszubildenden gedacht, nicht als Evaluationsinstrument durch die Mentoren/ Mentorinnen oder Ausbilder/Ausbilderinnen. Als eines der zentralen Ziele wird genannt, dass angehende Lehrkräfte ihr Wissen und ihre Kompetenzen reflektieren lernen sollen (vgl. Kelly \& Grenfell 2004). Darüber hinaus soll es als Unterstützung der Lehrenden in Ausbildung während der verschiedenen Ausbildungsphasen dienen, Anleitung zur Selbstbewertung und Anlass zu Gesprächen mit Ausbildnern/Ausbildnerinnen sowie Mentoren/Mentorinnen bieten.

Das EPOSTL, das bereits in zahlreichen Universitätsausbildungen oder in Teilen davon erfolgreich eingesetzt wird, ${ }^{5}$ ist damit ein Dokument, das Elemente der kompetenzorientierten Lehrerausbildung (CBTE-competence based teacher education) enthält (vgl. auch Grenfell 2012: 168) und diese mit dem zentralen Konzept des reflective practitioner-Modells zusammenführt, um eine fachspezifische Ausbildung im Bereich Fremdsprachen zu unterstützen. Trotz dieses hohen Anspruchs bleibt das EPOSTL mit seiner Engführung auf ein Fach und seiner nicht ganz überzeugenden Auswahl von Kompetenzbereichen hinter den Erwartungen an ein die Professionalität von Lehramtsstudierenden umfassend begleitendes Dokument zurück ${ }^{6}$. Es fehlt ihm zum einen an einer grundsätzlichen Auseinandersetzung mit der Lehrerpersönlichkeit und der Profession, zum anderen fehlen über die Kompetenzorientierung im Sinne von Handlungswissen (und dessen Reflexion) hinaus weite-

\footnotetext{
5 Zahlreiche Hochschulen insbesondere in Österreich haben das EPOSTL als Unterrichtsgegenstand oder auch als studienbegleitendes Instrument in der Lehramtsausbildung der ersten Phase verankert (vgl. Newby 2012), in Deutschland wird es bisher nicht so häufig studienbegleitend, sondern eher punktuell eingesetzt (vgl. Kupetz \& Ruhm 2012).

${ }^{6}$ Es fehlt beispielsweise der Kompetenzbereich Hör-/Sehverstehen ganz. Fiktionale Texte und Spielfilme werden nur wenig berücksichtigt.
} 
re fachliche und fachdidaktische Aspekte, wie die Selbstbewertung der Sprachpraxis, des Wissens und die differenzierte Reflexion von Lehr-/Lerntheorien und ihre historische Bedeutung in der fachdidaktischen Ausbildung sowie alle Bezüge zu Forschungsaspekten.7 Dabei wurde offensichtlich ein sehr wichtiger Faktor der Lehramtsausbildung (in den Fremdsprachen aber auch in anderen Fächern) vergessen oder hintangestellt. Die Fachdidaktiken der Hochschulen rekrutieren aus ihren Auszubildenden im Lehramt immer auch ihren wissenschaftlichen Nachwuchs, der sich am Ende des Lehramtsstudiums entscheidet, ob er eine Lehramtslaufbahn oder eine wissenschaftliche Karriere an der Hochschule mit einer Promotion einschlägt. Theoriebezogene Studieninhalte und Forschungsmethoden sowie Forschungsdesigns sind meistens bereits in die Lehramtsausbildung integriert und kommen dort während des gesamten Studiums zur Sprache. Dies geschieht, um den wissenschaftlichen Nachwuchs rechtzeitig auszubilden, aber auch um angehenden Lehrern/Lehrerinnen die Chance zu bieten, schon während der ersten Phase des Studiums die Lehr-/Lernsituation in ihren Fächern oder in allgemeinen pädagogischen Zusammenhängen selbst zu beforschen - etwa während einer Praktikumsphase oder auch für eine Staatsexamensarbeit oder MA-Thesis. Es werden im EPOSTL im Zielpunkt 7 zwar Forschungsprojekte genannt, die von den auszubildenden Sprachlehrenden durchgeführt werden sollen, ${ }^{8}$ Wissen (und damit Reflexion) über Forschungsprojekte wird aber nicht durch einen eigenen Kompetenzbereich innerhalb der Kann-Beschreibungen angesprochen, so dass es zweifelhaft bleibt, ob und woher die Studierenden dieses Wissen beziehen und wie sie es systematisch ausbauen sollen, um selbst zu forschen. Es ist aus der Sicht der ersten Ausbildungsphase von Lehrern/Lehrerinnen wenig angemessen, die zentralen Gebiete der qualitativen und quantitativen sowie hermeneutischen Forschung in den Kann-Beschreibungen auszusparen, bieten sie doch vielfältige Möglichkeiten und Anregungen zur weiteren Erkundung des Berufsfelds, stellen oft Grundlagen für Innovationen dar und laden Lehrer/Lehrerinnen letztlich auch zu action researchProjekten ein, die helfen können, Schwierigkeiten im Unterricht zu untersuchen, die eigene Rolle zu reflektieren und damit auch neu zu beleuchten. Lehrer/ Lehrerinnen, die oft Innovationen „von oben“ durchführen müssen, weil sich der Lehr-/Lernkontext stetig ändert, können diese Innovationen nur mit einer ausreichenden Wissensgrundlage in Lehr- und Lerntheorien beurteilen. Eine

\footnotetext{
${ }^{7} \mathrm{Zu}$ diesem Komplex gibt es zwei Deskriptoren im Kompetenzbereich Die Rolle des Fremdsprachenlehrenden: „Ich kann auf entsprechende Theorien bezüglich Sprache, Lernen, Kultur, etc. und auf relevante Forschungsergebnisse zurückgreifen, wenn ich meinen Unterricht gestalte.“ und „Ich kann relevante Artikel, Zeitschriften und Forschungsergebnisse in Bezug auf verschiedene Aspekte des Lehrens und Lernens finden." Diese stark verkürzten Darstellungen, die lerntheoretisches Wissen nur auf der Ebene der direkten Umsetzung in Unterricht abrufen, sind für eine reflektierende Lehrerpersönlichkeit kaum ausreichend.

${ }^{8}$ Im Dossier erscheint die Aufforderung, den Dokumenten auch Fallstudien und Aktionsforschungsbeispiele beizufügen, die dann reflektiert werden können, doch wird dieser Punkt in den KannBeschreibungen nicht ausreichend angeleitet.
} 
professionelle Beurteilung neuer Sachverhalte ist aber die Grundlage für eine professionelle Umsetzung in der täglichen Praxis. Auch jene Aspekte der Lehrerpersönlichkeit, die über rein fachliche und fachdidaktische Kompetenzen hinausgehen, z.B. persönliche Wertvorstellungen und Einstellungen werden im EPOSTL nicht reflektiert. So kann das Portfolio für angehende Englischlehrkräfte zwar einen gewissen Rahmen vorgeben für die Lehramtsausbildung von Sprachenlehrern/-lehrerinnen, dieser Rahmen ist aber nicht ausreichend breit an den tatsächlichen Ausbildungskomponenten und den heutigen Anforderungen an Lehrer/Lehrerinnen orientiert.

Darüber hinaus bietet das EPOSTL auch keinen umfassenden, phasenübergreifenden Ansatz an, der die Ausbildung der Lehramtspersönlichkeit insgesamt begleiten könnte. Dies gilt insbesondere für die deutsche Lehramtsausbildung, die - so unterschiedlich sie in der föderalistischen Bildungsstruktur auch sein mag immer zwei oder drei Fachdidaktiken, zwei oder drei Fachwissenschaften ${ }^{9}$ und die Grundwissenschaften umfasst. Sollte es politischer Wille sein, die Lehramtsausbildung durch ein Portfolio zu begleiten, dann wäre es sicher vorteilhaft, wenn ein phasenübergreifendes Lehramtsportfolio alle diese Teilbereiche der Ausbildung zusammenfassen und durch ein ausdifferenziertes Modell der Lehrerpersönlichkeit sowie der beruflichen und persönlichen Reflexion miteinander verbinden könnte.

\section{Vom reflective practitioner zu neuen Leitbildern der Lehrerausbildung}

Hatties Studie zum guten Unterricht (2013) ${ }^{10}$ hat durch ihre Datenvielfalt eindrucksvoll unter Beweis gestellt, wie wichtig gute Lehrer/Lehrerinnen für ein Gelingen des Unterrichts sind. Allerdings beschäftigt sich die Studie nicht damit, wie man solch erfolgreiche Lehrkräfte ausbilden kann und mit welchen pädagogischen, fachdidaktischen, fachwissenschaftlichen Wissensbeständen und welchen Persönlichkeitsmerkmalen sie in einzelnen Schulkontexten und Fächern ausgestattet sein sollten, um erfolgreichen Fachunterricht zu ermöglichen. Um einer Antwort auf diese Frage näher zu kommen, ist es nötig, sich mit anderen Studien zu beschäftigen, die - meist aus der Perspektive der pädagogischen Psychologie oder Pädagogik - Konzepte von guten Lehrkräften erstellen. Mehrere solcher Modelle, die in den letzten fünfzehn Jahren erstellt wurden, gehen davon aus, dass zu einer guten Lehrkraft weitaus mehr gehört als Kompetenzen; sie vereinen

\footnotetext{
${ }^{9}$ Drei Fachwissenschaften und -didaktiken sind in der Regel für die angehenden Grundschullehrkräfte Pflicht.

${ }^{10}$ Der neuseeländische Bildungsforscher John Hattie hat in einer Studie mit mehr als 800 Metaanalysen, die wiederum 50000 Einzelstudien zusammenfassen, untersucht, was guten Unterricht ausmacht. Insgesamt waren an den Untersuchungen 250 Millionen Schüler/Schülerinnen beteiligt. Sein Buch Visible Learning for Teachers (2013) liefert die umfangreichste Darstellung der weltweiten Unterrichtsforschung. Hattie verbreitert seine Datenbasis ständig mit neuen Erhebungen.
} 
persönlichkeitsorientierte und kompetenzorientierte Anforderungen miteinander und betonen auch, dass es nicht nur ein normativ ausgerichtetes Kompetenzmodell für gute Lehrkräfte geben kann, da jeweils unterschiedliche Kontexte für Lehrer/ Lehrerinnen (etwa Schulform, Fachunterricht etc.) eine größere Detailgenauigkeit bei den Merkmalen erfordern. Deshalb stellen sie meist Referenzrahmen vor, innerhalb derer die Anforderungen an gute Lehrkräfte definiert werden. Korthagen präsentiert bereits 2004 sein „Zwiebelmodell“ einer Lehrerpersönlichkeit, in dem im wahrsten Sinne des Wortes Schicht um Schicht die professionelle Persönlichkeit dargestellt wird.

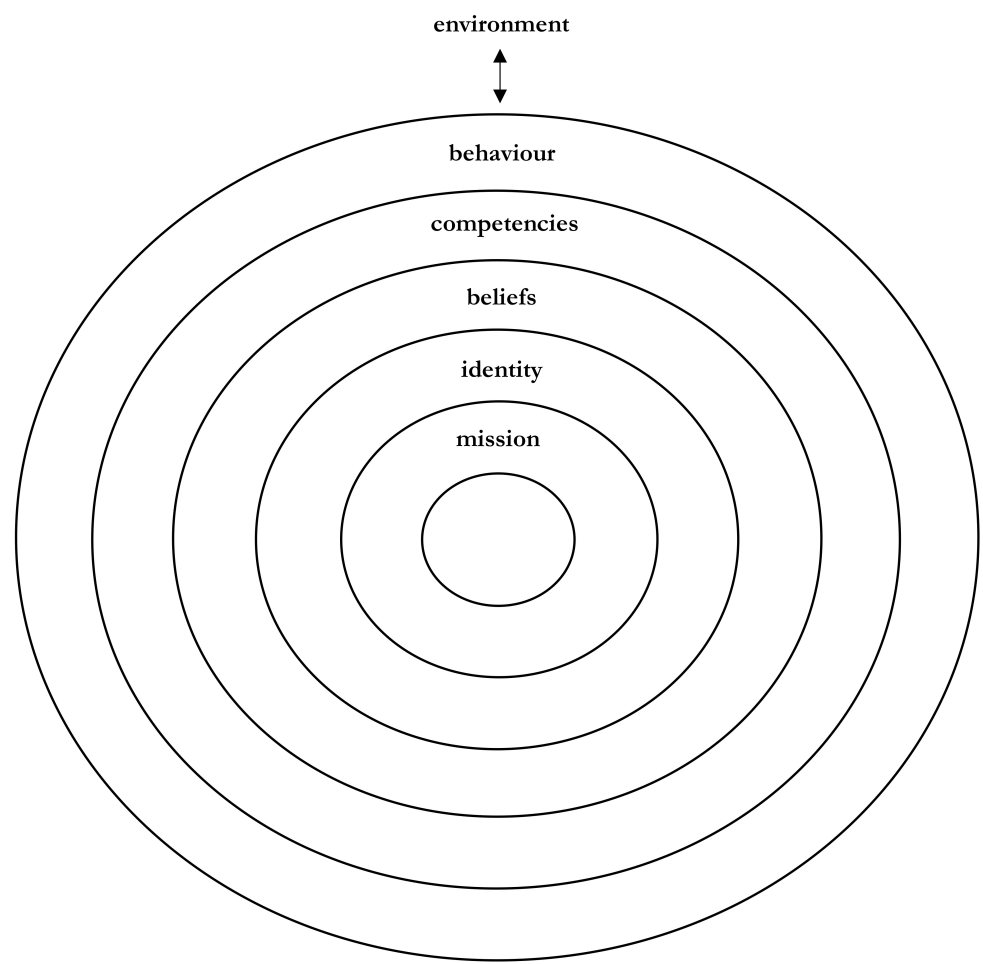

Abb. 1: Das Zwiebelmodell der Lehrerpersönlichkeit nach Korthagen (2004)

Die einzelnen Schichten der Zwiebel beeinflussen sich von innen nach außen und umgekehrt von außen nach innen (vgl. Korthagen 2004: 80). Die äußere Schicht beschreibt bei Korthagen die konkrete Umgebung der Lehrkraft. Durch sein/ihr Lehrerverhalten reagiert der Lehrer/die Lehrerin auf diese Umgebung; in der Lehrerausbildung ist dies die zweite Schicht, eben jene, die meist im Mittelpunkt der Aufmerksamkeit der Lehramtsstudierenden steht, sie wollen wissen, wie man erfolgreich mit und vor einer Klasse agiert. Bestimmt wird das Lehrerhandeln nach 
Korthagen von den Kompetenzen des Lehrers/der Lehrerin, die Wissen und Fertigkeiten wie auch Einstellungen beinhalten (vgl. hierzu Stoof et al. 2000 in Korthagen 2004: 80). Korthagen betont, dass diese Schicht das Lehrerverhalten als Potenzial, nicht als tatsächliches beobachtbares Verhalten umfasst, da es situationsabhängig ist. Beeinflusst wird das Lehrerverhalten durch pädagogische und fachdidaktische Konzepte und Annahmen. Diese sind zum Teil durch Erfahrungen als Schüler/Schülerin geprägt, zum Teil durch die Ausbildung erworben. Korthagen hebt in diesem Zusammenhang hervor, dass vor allem die alten, auf Eigenerfahrung beruhenden Konzepte oft überdauern in einer Lehrerpersönlichkeit (vgl. Korthagen 2004: 81). Die Konzepte gründen sich seines Erachtens vor allem auf die professionelle Identität der Lehrkraft, die der Frage nachgeht „Wer bin ich als Lehrer/Lehrerin?"/,Wie sehe ich meine Rolle?" Diese Identität stellt die fünfte Zwiebelschicht dar, hier nur mit identity bezeichnet. Die sechste, darunterliegende Schicht wird als Mission gekennzeichnet und meint die Motive, aus denen heraus sich die Selbstkonzepte der Lehrkraft speisen, laut Korthagen (2004: 85) eine spirituelle oder transpersonelle Ebene.

Where the identity level is concerned with the personal singularity of the individual, the spirituality level is about "the experience of being part of meaningful wholes and in harmony with superindividual units such as family, social group" (Boucouvalas, 1988). [...] For teachers, we can think of ideals such as creating more acceptance of differences between people creating feelings of self-worth in children, and so forth. In any case, we are talking about deeply felt, personal values that the person regards as inextricably bound up with his or her existence. People are not always equally aware of this level of themselves (Korthagen 2004: 86).

Kohonen, der das Modell aufgreift und für den Fremdsprachenunterricht umbaut, bescheinigt Korthagen Unsicherheit und Schwierigkeiten bei der Definition dieser Sphären. Er bietet in seinem Aufsatz zur Lernerautonomie, der sich auch intensiv mit der Rolle der Lehrkraft auseinandersetzt, ein zweites Zwiebelmodell an, das zum einen die äußere Rahmung (environment) weglässt, zum anderen die ganz innersten Schichten neu definiert (vgl. Kohonen 2012: 32-34):

Korthagen also seems to have difficulty in distinguishing the two innermost levels from each other and from the teacher's personal identity as a human being. I therefore decided to add the teacher's personal identity (conception of man, Who am I as a person?') as the innermost level in the model ... Thus, professional identity could answer the question of identity as an educator (,Who am I as a teacher?') while mission could be related to the educational values and purposes of the professional calling (Why am I in the classroom?) (Kohonen 2012: 34). 
Trotz dieser Einwände bleiben die Grundelemente des Zwiebelmodells bestehen; Kohonen transferiert sie aber explizit auf den Kontext des Fremdsprachenlehrers/ der Fremdsprachenlehrerin. Er zeigt im Folgenden auf, wie die flacheren und tieferen Strukturen der Lehrerpersönlichkeit (,shallow and deep structures"; Kohonen 2012: 32-34) im Fremdsprachenunterricht beim Erziehen zum autonomen Lerner/zur autonomen Lernerin beitragen können, wobei er mit flacheren Strukturen das Lehrerverhalten im Klassenzimmer meint, während alle anderen Schichten zu den tieferen Strukturen gezählt werden.

Korthagens's model of the teacher's professional growth underscores the importance of educational values for being a language educator. It also suggests a reciprocal relationship between the shallow structures of organizing classroom work and the deep structures of the ethical underpinnings of teaching. Working on this interplay between personal identity, educational mission and classroom practices provides a continuing source of inspiration for the teacher's professional growth (Kohonen 2012: 34).

\section{Ein neues Modell der Lehrerpersönlichkeit für Ausbildungs- und Weiterbildungskontexte}

Auch im deutschen Ausbildungskontext für Lehramtsstudierende bzw. Fremdsprachenlehrende kann dieses umfassende Modell als eine gute Grundlage für differenzierte Lernziele, für Lehramtscurricula und Ausbildungsgänge für die Fremdsprachenlehrenden in den verschiedenen Schularten dienen. Das folgend abgebildete Modell stellt eine Weiterentwicklung der Modelle von Korthagen und Kohonen dar, das für einen deutschen Ausbildungskontext in diesem Artikel, insbesondere für Fremdsprachenlehrkräfte, intendiert ist. Es kann natürlich auch für andere fachdidaktische Kontexte genutzt werden. 


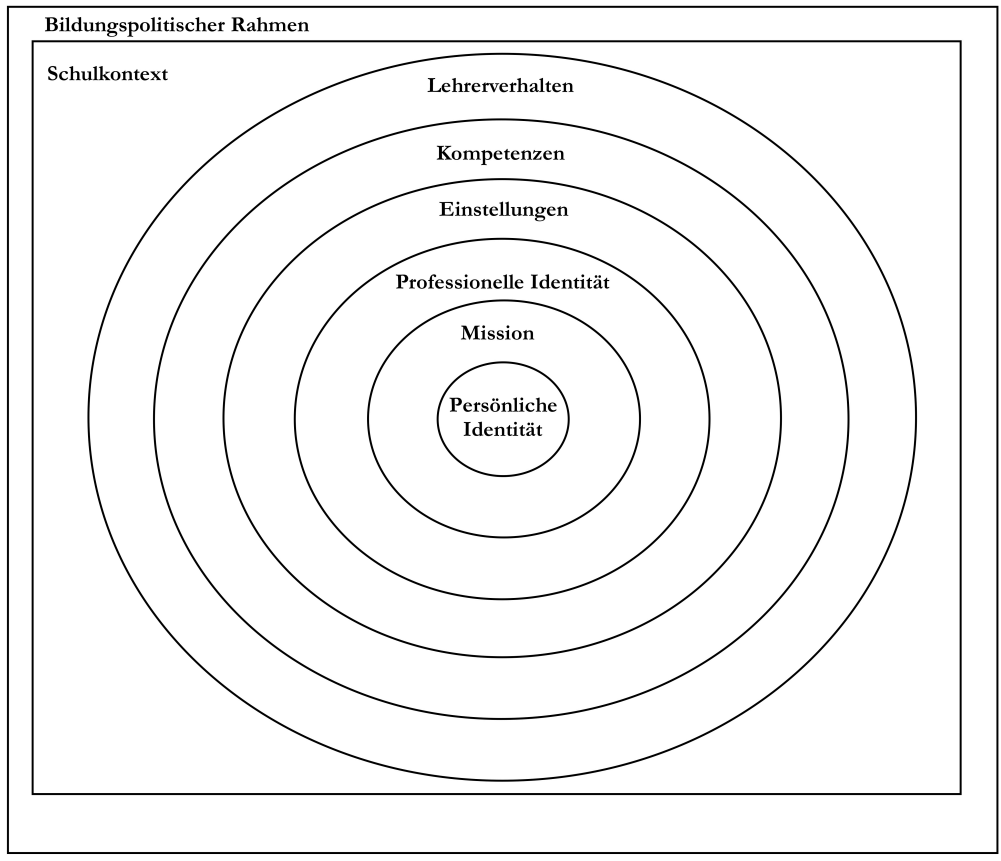

Abb. 2: Die Lehrerpersönlichkeit als Modell

Was bei Korthagen als pädagogisches Modell für Lehrer/Lehrerinnen gedacht war, soll hier alle Fächer des angehenden Lehrers/der angehenden Lehrerin umfassen, um in einem deutschen Bildungskontext (für eine Zwei-Fach- oder Drei-FachLehrkraft mit grundwissenschaftlicher Ausbildung) einsetzbar zu sein. In der hier vorgestellten Fassung wird das Zwiebelmodell von zwei Quadraten umgeben, dessen äußeres die bildungspolitischen Rahmenbedingungen abbilden soll. Das innere Quadrat stellt den jeweiligen Schulkontext dar. Diese beiden außerhalb des Zwiebelmodells liegenden Bedingungsfaktoren sind so weder bei Korthagen noch bei Kohonen vorhanden, symbolisieren aber unmissverständlich, dass die Lehramtsausbildung und hier ganz speziell die Fremdsprachenlehrerausbildung nicht im „luftleeren Raum“ stattfindet und bildungspolitische Vorgaben oder spezifische schulische Ansprüche ihren Einfluss stets durch institutionelle Bedingungen (Gesetze, Erlasse etc.) und durch Vertreter/Vertreterinnen der Institutionen (Ausbildende, Mentoren/Mentorinnen, aber dann auch Schüler/Schülerinnen) geltend machen.

Da die Schwierigkeit für auszubildende Lehrkräfte in der Regel darin liegt, ein solches Modell auf sich selbst zu beziehen, d.h. die einzelnen „Zwiebelringe“ in Bezug auf sich selbst zu reflektieren, werden im folgenden Fragen mit den einzelnen Aspekten des Modells verknüpft, die darauf abzielen, deutlich zu machen, welche Aspekte der Lehrerpersönlichkeit jeweils gemeint sind. Die unten abgebildete Tabelle zeigt solche Fragen, kann jedoch jeweils nur eine Auswahl vorstellen, 
die ergänzt werden kann. Hervorzuheben ist hier noch, dass sich alle abgebildeten Fragen auf die drei an der Lehrerausbildung beteiligten Bereiche Fachdidaktik, Fachwissenschaft und Pädagogik beziehen.

Tab. 1: Reflexionsfragen zum Modell der Lehrerpersönlichkeit (nach Kohonen 2012)

\begin{tabular}{|l|l|l|}
\hline Grafikelement & \multicolumn{1}{|c|}{ Titel } & \multicolumn{1}{c|}{ Fragestellung } \\
\hline $\begin{array}{l}\text { äußeres } \\
\text { Quadrat }\end{array}$ & $\begin{array}{l}\text { Bildungspolitischer } \\
\text { Rahmen }\end{array}$ & $\begin{array}{l}\text { In welchem gesetzlichen und } \\
\text { bildungspolitischen Rahmen agiere ich? } \\
\text { Kenne ich mich in diesem Rahmen aus? }\end{array}$ \\
\hline $\begin{array}{l}\text { inneres } \\
\text { Quadrat }\end{array}$ & Schulkontext & $\begin{array}{l}\text { In welchen Schulformen und Fächern } \\
\text { agiere ich? In welchem schulischen } \\
\text { Kontext agiere ich? }\end{array}$ \\
\hline 2. Ring & Lehrerverhalten & $\begin{array}{l}\text { Wie agiere ich in den unterschiedlichen } \\
\text { Kontexten des schulischen Handelns } \\
\text { (Klassenzimmer, Lehrergespräch, } \\
\text { Kooperation mit Kollegen etc.)? }\end{array}$ \\
\hline 3. Ring & Kompetenzen & $\begin{array}{l}\text { Welche Wissensbestände und Fertigkeiten } \\
\text { habe ich? Wo sehe ich Defizite? }\end{array}$ \\
\hline 4. Ring & Einstellungen & $\begin{array}{l}\text { Welche professionellen Einstellungen } \\
\text { habe ich? Wo erkenne ich fachdidaktische } \\
\text { Probleme? }\end{array}$ \\
\hline 5. Ring & $\begin{array}{l}\text { Mission: Persönliche } \\
\text { Überzeugungen und } \\
\text { Anliegen im } \\
\text { gesellschaftlichen Leben }\end{array}$ & $\begin{array}{l}\text { Weshalb bin ich in der Schule, in der } \\
\text { Klasse, im Fremdsprachenunterricht? }\end{array}$ \\
\hline
\end{tabular}

Das hier vorgestellte Modell ist - anders als die zwei zitierten Modelle von Korthagen und Kohonen - für die Ausbildung und für die berufliche Weiterentwicklung von Lehramtsstudierenden unter Bezugnahme auf ihre grundwissenschaftlichen, fachwissenschaftlichen und fachdidaktischen Ausbildungsverläufe gedacht. Es möchte damit zwei ganz grundlegende Ziele anstreben: Erstens soll sich der/die Lehramtsstudierende, der/die dieses Modell betrachtet, über die Multiperspektivität des angestrebten Berufs klarwerden. Es soll so schon frühzeitig eine deutliche Verortung der Lehrerpersönlichkeit innerhalb der bildungspolitischen, schulischen, pädagogischen und fachdidaktischen Systeme erfolgen. Zweitens soll der/die reflektierende Studierende sich darüber klarwerden, welche Strukturen und Schichten zu einer Lehrerpersönlichkeit gehören, so dass frühzeitig erkannt wird, wie neben pädagogischen Aspekten auch fachwissenschaftliche und fachdidakti- 
sche Seiten des Berufs entwickelt bzw. weiterentwickelt werden müssen. Das Modell hilft deshalb kleinschrittig nachzufragen, wie sich jede/r einzelne Studierende der Lehramtsfächer selbst verortet und einschätzt, damit alle Schichten voneinander unterschieden werden können. Es strebt auch an, dass der/die sich selbst reflektierende Lehramtsstudent/-studentin oder der/die sich selbst beobachtende Lehrer/Lehrerin bei der Reflexion nicht nur positive Aspekte herausarbeiten kann, sondern auch Schwächen und Defizite zu erkennen vermag. Erst beides zusammen stärkt die Selbstwirksamkeit und macht eine Selbstbetrachtung in Ausbildung und Weiterbildung sinnvoll.

Das Zwiebelmodell in der vorliegenden Form ist seit dem Wintersemester 2017/18 an der Justus-Liebig-Universität in Gießen versuchsweise Bestandteil der englischdidaktischen Lehramtsausbildung im ersten Semester und in der Praktikumsvorbereitung. Es wird mit den dazugehörigen Fragen eingeführt und auf alle fachdidaktischen Themen (z.B. Lerntheorien, interkulturelles Lernen, Kompetenzen im Englischunterricht, Fragen des Curriculums, Themen der Inklusion) angewandt. Das bedeutet, dass die Lehramtsstudierenden im Laufe des ersten Semesters jeweils reflektieren, ob und in welchen „Schichten“ das jeweilige Thema für sie als Lehramtsstudierende der Englischdidaktik relevant ist und mit welchen Fragen der Lehrerprofessionalität es sich verbinden lässt. Da das Zwiebelmodell wöchentlich in der Einführungsveranstaltung thematisiert wird, kann man sich erhoffen, dass den Studierenden eine Reflexion ihrer eigenen Professionalisierung und ihrer persönlichen Einstellungen und Kompetenzen in Bezug auf ihren zukünftigen Beruf und ihre Ausbildung erleichtert wird. ${ }^{11}$ Feststellen lässt sich bereits jetzt, dass der Fragenkatalog, der natürlich nicht erschöpfend ist, aber einen Anreiz zur Operationalisierung des Zwiebelmodells gibt, eine wichtige Hilfe, eine Art scaffolding für die Lehramtsstudierenden darstellt, die es insbesondere im ersten Studiensemester meist als schwierig empfinden, den Lehrberuf und ihre Ausbildung in der fachdidaktischen Ausprägung zu reflektieren.

\section{Das Modell zur Lehrerpersönlichkeit als Reflexionsgrundlage für ein phasenübergreifendes grundwissenschaftlich, fachwissenschaftlich und fachdidaktisch ausgerichtetes Lehramtsportfolio}

Wenn man davon ausgeht, dass ein Portfoliomodell die angehenden Fremdsprachenlehrkräfte über den gesamten Studien- und Ausbildungsprozess hindurch und darüber hinaus begleiten soll, muss es, um ernst genommen zu werden, in die hochschuldidaktischen und schulischen Lehr-/Lernsituationen fest integriert und

\footnotetext{
${ }^{11}$ Das Modell ist im WiSe 2017/18 zum ersten Mal durch Leitfaden-Interviews mit einer Auswahl von Erstsemester-Studierenden erprobt worden. Weitere empirische Untersuchungen folgen in den nächsten Semestern.
} 
auch als Prüfungsinhalt eingesetzt werden. Es muss sich in mehrere leicht zu handhabende Teile zerlegen lassen, die möglichst als E-Portfolio ohne den Aufwand einer Druckfassung unterschiedlich genutzt werden. In Anlehnung an bereits bekannte Portfoliostrukturen könnte es aus vier Teilen mit unterschiedlichen Funktionen bestehen.

Der Lehramtspass dokumentiert summativ mit der Sammlung von Zeugnissen, Sprachqualifikationen und Belegen über Auslandsaufenthalte des Portfolioeigners/ der Portfolioeignerin, welche institutionellen Schritte beim Lehramtsstudium (z.B. beim Erlernen der Sprachen) bereits absolviert wurden. Dabei werden bei Fremdsprachenlehrkräften etwa die Mehrsprachigkeit der Portfolioeigner/-eignerinnen, sowie ihre interkulturellen Erfahrungen sichtbar gemacht. Dieser Teil des Portfolios hat Dokumentationsfunktion, d.h. er ist bei Wechseln in eine andere schulische oder universitäre Bildungsinstitution und bei beruflichen Bewerbungen im In- und Ausland von großem Wert.

Die Selbsteinschätzung (mit Könnens-Beschreibungen) hat eine prozessdiagnostische und eine planerische Funktion: Hier findet die angehende Lehrkraft Deskriptoren zu den individuell wichtigen Kompetenzbereichen. Vorstellbar sind in diesem Kontext Deskriptoren zu den drei großen Studienbereichen Fachwissenschaft, Fachdidaktik und Grundwissenschaften, die in Illustration 4 dargestellt sind. Durch die individuelle Beschreibung des eigenen Kompetenzstandes mit Hilfe der Checklisten wird ein Selbstevaluationsprozess in Gang gesetzt, der Lernenden abverlangt, ihre Lernfortschritte in regelmäßigen Abständen differenziert einzuschätzen. Die Checklisten beziehen sich grundsätzlich immer auf alle vom Lernenden gesprochenen, erworbenen oder formell erlernten Kompetenzbereiche und werden für jeden gesondert ausgefüllt.

Im Dossier, dem dritten Teil, kommen sowohl die produktorientierte Dokumentationsfunktion wie auch die prozessdiagnostische Funktion zum Tragen, es ist ebenfalls eine formative Art der Selbsteinschätzung: Indem die Portfolioeigner/ -eignerinnen selbst besonders gute, jeweils aktuelle Arbeiten zum Nachweis ihres professionellen Kompetenzstandes auswählen und dort präsentieren, akkumulieren sich im Laufe der Zeit Dokumente ihres Lernprozesses, die einen Lernfortschritt belegen. Auch die Auswahl der einzelnen Arbeitsbeispiele regt zur Reflexion über den eigenen Lernprozess an. Dieser Teil des Portfolios ist für alle Ausbilder/Ausbilderinnen einsehbar und wird auch als Grundlage für mündliche Prüfungsteile in den zwei Staatsexamina oder den Bachelor- und Master-Prüfungen genutzt. Später dient das Dossier als Dokumentation der erworbenen Kompetenzen bei Bewerbungen, Einstellungsgesprächen oder Jahresgesprächen mit der Schulleitung. 


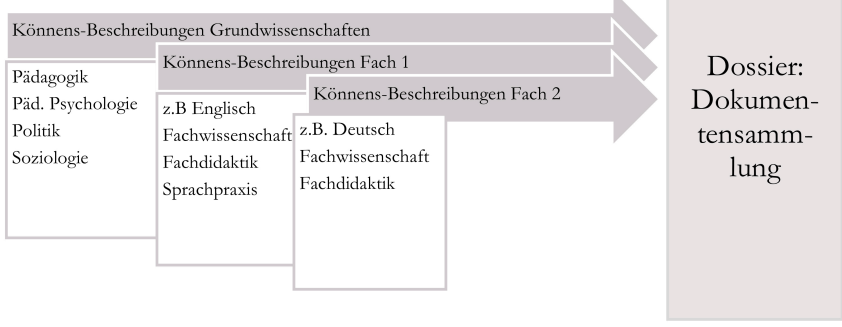

Reflexion von Wissen

Erfahrung und

Selbsteinschätzung

in einem

Fach und darüber hinaus

Abb. 3: Verhältnis von Kann-Beschreibungen und Reflexion

Als vierter und letzter Teil des Portfolios ist der Reflexionsteil zu nennen, der ebenfalls eine prozessdiagnostische Funktion innehat. Er ist in der Regel nicht einsehbar für die Ausbilder/Ausbilderinnen, kann aber, falls dies gewünscht wird, in bestimmten Phasen, z.B. den Praktikumsphasen des Studiums oder im Referendariat, die zentralen Gelenkstellen der Ausbildung darstellen, als Grundlage für Gespräche sichtbar gemacht werden. Der Reflexionsteil verbindet alle Teile des Portfolios miteinander, dort wird der Transfer von Wissen und Erfahrung in allen Fächern und über die Fächer hinaus stattfinden, der Grundlage der Lehrerpersönlichkeit ist.

Tab. 2: Mögliche Nutzung des Lehramtsportfolios in der zweiphasigen Ausbildung und danach (kursiv: nicht oder nur freiwillig sichtbar gemachte Teile und Leistungen im Portfolio)

\begin{tabular}{|l|l|l|l|}
\hline Phase & \multicolumn{1}{|c|}{$\begin{array}{c}\text { Ausbildungsab- } \\
\text { schnitt }\end{array}$} & \multicolumn{1}{|c|}{ Portfolioteil } & \multicolumn{1}{c|}{ Portfoliofunktion } \\
\hline & 1. Semester & Einführung & $\begin{array}{l}\text { Vorbereitung auf das } \\
\text { Portfolio, } \\
\text { Anleitung zur Reflexion }\end{array}$ \\
\hline & $\begin{array}{l}\text { Grundstudium } \\
\text { ab dem 2. } \\
\text { Semester }\end{array}$ & $\begin{array}{l}\text { Lehramtspass } \\
\text { Selbstbeurteilung } \\
\text { Reflexionsteil } \\
\text { Dossier }\end{array}$ & $\begin{array}{l}\text { Sammeln von offiziellen } \\
\text { Dokumenten etc. } \\
\text { Einschätzung der professionellen } \\
\text { Kompetenz } \\
\text { Reflexion und Transfer } \\
\text { Dokumentation von } \\
\text { Leistungen } \\
\text { (z.B. Seminararbeiten) }\end{array}$ \\
\hline
\end{tabular}




\begin{tabular}{|c|c|c|c|}
\hline Phase & $\begin{array}{l}\text { Ausbildungsab- } \\
\text { schnitt }\end{array}$ & Portfolioteil & Portfoliofunktion \\
\hline & $\begin{array}{l}\text { Pädagogisches } \\
\text { Praktikum }\end{array}$ & $\begin{array}{l}\text { Lehramtspass } \\
\text { Selbstbeurteilung } \\
\text { Reflexionsteil } \\
\text { Dossier }\end{array}$ & $\begin{array}{l}\text { Sammeln von offiziellen } \\
\text { Dokumenten } \\
\text { Einschätzung der professionellen } \\
\text { Kompeten₹. } \\
\text { Reflexion und Transfer } \\
\text { Dokumentation von } \\
\text { Leistungen } \\
\text { (z.B. Unterrichtsentwürfe, } \\
\text { Videoaufzeichnungen von } \\
\text { eigenem Unterricht etc.) }\end{array}$ \\
\hline & Hauptstudium & $\begin{array}{l}\text { Lehramtspass } \\
\text { Selbstbeurteilung } \\
\text { Reflexionsteil } \\
\text { Dossier }\end{array}$ & $\begin{array}{l}\text { Sammeln von offiziellen } \\
\text { Dokumenten } \\
\text { Einschätzung der professionellen } \\
\text { Kompetenz. } \\
\text { Reflexion und Transfer } \\
\text { Dokumentation von } \\
\text { Leistungen } \\
\text { (z.B. Seminararbeiten, } \\
\text { Workshop-Produkte etc.) }\end{array}$ \\
\hline & 1. Staatsexamen & $\begin{array}{l}\text { Lehramtspass } \\
\text { Dossier }\end{array}$ & $\begin{array}{l}\text { Lehramtspass und Dossier } \\
\text { bilden Grundlage für einen } \\
\text { Pflichtteil der mündlichen } \\
\text { Prüfung }\end{array}$ \\
\hline & $\begin{array}{l}\text { Beginn } \\
\text { Referendariat }\end{array}$ & $\begin{array}{l}\text { Lehramtspass } \\
\text { Dossier }\end{array}$ & $\begin{array}{l}\text { Lehramtspass und Dossier } \\
\text { geben Aufschluss über } \\
\text { offizielle Zeugnisse und } \\
\text { Studienleistungen }\end{array}$ \\
\hline & Referendariat & $\begin{array}{l}\text { Lehramtspass } \\
\text { Selbstbeurteilung } \\
\text { Reflexionsteil } \\
\text { Dossier }\end{array}$ & $\begin{array}{l}\text { Sammeln von offiziellen } \\
\text { Dokumenten } \\
\text { Einschätzung der professionellen } \\
\text { Kompetenz. } \\
\text { Reflexion und Transfer } \\
\text { Dokumentation von } \\
\text { Leistungen (z.B. } \\
\text { Unterrichtsplanungen, } \\
\text { Reflexion von Unterricht) }\end{array}$ \\
\hline
\end{tabular}




\begin{tabular}{|c|c|c|c|}
\hline Phase & $\begin{array}{l}\text { Ausbildungsab- } \\
\text { schnitt }\end{array}$ & Portfolioteil & Portfoliofunktion \\
\hline & 2. Staatsexamen & $\begin{array}{l}\text { Lehramtspass } \\
\text { Dossier }\end{array}$ & $\begin{array}{l}\text { Lehramtspass und Dossier } \\
\text { bilden Grundlage für einen } \\
\text { Pflichtteil der mündlichen } \\
\text { Prüfung }\end{array}$ \\
\hline & $\begin{array}{l}\text { Bewerbungsphase } \\
\text { und Einstellung }\end{array}$ & $\begin{array}{l}\text { Lehramtspass } \\
\text { Dossier }\end{array}$ & $\begin{array}{l}\text { Lehramtspass und Dossier } \\
\text { bilden Bestandteile für die } \\
\text { Bewerbung und das } \\
\text { Einstellungsgespräch }\end{array}$ \\
\hline & $\begin{array}{l}\text { Berufliche } \\
\text { Tätigkeit }\end{array}$ & $\begin{array}{l}\text { Lehramtspass } \\
\text { Dossier }\end{array}$ & $\begin{array}{l}\text { Lehramtspass wird weiter } \\
\text { ergänzt } \\
\text { Dossier wird weiter ergänzt } \\
\text { (z.B. mit besonderen } \\
\text { Unterrichtseinheiten, } \\
\text { Fortbildungen, etc.) }\end{array}$ \\
\hline & $\begin{array}{l}\text { Jährliche } \\
\text { Schulleiter- } \\
\text { gespräche }\end{array}$ & $\begin{array}{l}\text { Lehramtspass } \\
\text { Dossier }\end{array}$ & $\begin{array}{l}\text { Lehramtspass und Dossier } \\
\text { bilden Bestandteil für das } \\
\text { Gespräch }\end{array}$ \\
\hline & $\begin{array}{l}\text { Beförderungen } \\
\text { oder } \\
\text { Schulwechsel }\end{array}$ & $\begin{array}{l}\text { Lehramtspass } \\
\text { Dossier }\end{array}$ & $\begin{array}{l}\text { Lehramtspass und Dossier } \\
\text { bilden Grundlage für weitere } \\
\text { Bewerbungen oder } \\
\text { Beförderungsgespräche }\end{array}$ \\
\hline \multicolumn{4}{|c|}{ oder } \\
\hline & $\begin{array}{l}\text { Evtl. } \\
\text { Qualifikations- } \\
\text { phase als } \\
\text { Doktorandin/ } \\
\text { Doktorand }\end{array}$ & $\begin{array}{l}\text { Lehramtspass } \\
\text { Selbstbeurteilung, } \\
\text { Reflexionsteil } \\
\text { Dossier }\end{array}$ & $\begin{array}{l}\text { Sammeln von offiziellen } \\
\text { Dokumenten } \\
\text { Einschätzung der professionellen } \\
\text { Kompetenz } \\
\text { Reflexion als Grundlage für } \\
\text { Forschungsarbeiten } \\
\text { Dokumentation von } \\
\text { Leistungen (z.B. } \\
\text { Unterrichtsbeobachtungen, } \\
\text { Reflexion von Unterricht) }\end{array}$ \\
\hline
\end{tabular}

In der Tabelle wird gezeigt, wie ein solches Portfolio in die unterschiedlichen Phasen der Lehramtsausbildung und danach integriert werden kann. Die kursiv markierten Portfolioteile sind nur für den Portfolioeigentümer/die Portfolioeigentümerin bestimmt, Lehramtspass und Dossier dagegen sind Portfolioteile, die stets auch öffentlich genutzt werden. Der Reflexionsteil hat zwei Funktionen: Er ist zum einen und in der Regel ein privates Dokument, kann aber zum anderen auch 
als Gesprächsanlass in der Ausbildung, z.B. nach den Praktika, genutzt werden, denn gerade in der ersten Phase der Ausbildung sollte gelegentlich überprüft werden, ob ausreichend und detailliert genug reflektiert wird. Durch diesen Portfolioteil können sich spannende Gespräche zwischen den angehenden Lehrern/ Lehrerinnen in Seminaren, aber auch zwischen auszubildender Lehrkraft und Mentoren/Mentorinnen oder Dozenten/Dozentinnen ergeben.

Der Portfolioteil zur Selbstbeurteilung bleibt stets ein privates, nicht öffentliches Dokument, das zwar in die Reflexion einfließt, aber nicht an Ausbilder/ Ausbilderinnen weitergegeben wird. Vorstellbar sind folgende Kompetenzfelder, die sich auf die Kompetenzbereiche des EPOSTL stützen, aber auch an einigen Punkten weit darüber hinausgehen:

1. Lehr-/Lernkontexte

2. Lerntheoretische Grundlagen

3. Forschungsgrundlagen

4. Methodische Aspekte

5. Unterrichtsplanung

6. Unterrichtsdurchführung

7. Beurteilung der Lernfortschritte

8. Weitere Rollen der Lehrkraft im jeweiligen Lehr-/Lernkontext (Elternberatung, Kooperation mit Kollegen und Kolleginnen sowie mit pädagogischem Personal, Kooperation mit Ämtern etc.)

9. Sprachenkompetenz und interkulturelle Kompetenz

Dieser Vorschlag gründet sich auf Erfahrungen in der englischen Fachdidaktik; er mag in anderen Fachdidaktiken oder auch aus Sicht der Grundwissenschaften noch erweiterungsbedürftig sein.

\section{$8 \quad$ Fazit}

Die hier dargestellten Vorüberlegungen für ein phasenübergreifendes Lehramtsportfolio haben die heutigen Grundlagen des Lehramtsstudiums in ihrer föderalen Diversität zu beschreiben versucht und daraus Schlussfolgerungen für die Ausbildung zukünftiger Lehrer/Lehrerinnen gezogen. Dabei lassen sich folgende Schlussfolgerungen zusammenfassen:

1. Ein Lehramtsportfolio für ausschließlich ein Fach oder einen Teil eines Faches, z.B. die Englischdidaktik, ergibt in der Ausbildungsstruktur für Lehramtsstudierende in Deutschland wenig Sinn. Es wird bei den unterschiedlichen Stakeholdern - wie bereits geschildert (vgl. Neß 2013) - auf wenig Akzeptanz stoßen. Nur ein alle Ausbildungsfächer und -bereiche bündelndes Portfolio rechtfertigt die große Anstrengung, alle Kompetenzen, die in den Fächern ausgebildet werden, durch Deskriptoren abzubilden. 
2. Ein phasenübergreifendes Lehramtsportfolio, das ist deutlich geworden, benötigt Akzeptanz von den Beteiligten, da sie ihre Ausbildung auf ein solches Portfolio ausrichten müssen. Die Standards, die durch die Deskriptoren beschrieben werden, müssen verpflichtend für eine Ausbildung sein, sonst ist ein solches Portfolio wenig sinnvoll. Diese Verpflichtung ist für manche Bereiche der Ausbildung, besonders in der ersten Phase für Hochschulen, ungewohnt und scheint zunächst der oft zitierten Freiheit für die Lehre zu widersprechen. Tatsächlich würde eine solche Verpflichtung aber der Ausbildung der Lehramtsstudierenden guttun, denn sie würde als Qualitätskontrolle eine solide Breite ihrer Kompetenzen in allen beteiligten Fächern garantieren. Dies würde nicht nur den zukünftigen Schülern/Schülerinnen zu Gute kommen, sondern auch bis in die Besetzungspolitik der Hochschulen wirken, die ein ausreichend breites Lehrangebot vorhalten müssten. Für die Fremdsprachen und ihre Didaktik bedeutet dies sehr konkret, dass spezielle Dozenten/Dozentinnen für jede Fremdsprache und Fremdsprachendidaktiker/-didaktikerinnen mit einer grundständigen Ausbildung in Didaktik eingestellt werden müssten. Nicht immer haben deutsche Universitäten eine solche Besetzungspolitik in den letzten zwanzig Jahren verfolgt.

3. Ein phasenübergreifendes Lehramtsportfolio benötigt ein Modell der Lehrerpersönlichkeit, das einen Konsens herstellt zwischen den Fachwissenschaften, den Fachdidaktiken und den Grundwissenschaften. Es sollte die politischen, kulturellen und professionellen Aspekte des Lehrerberufs und seine gesellschaftliche Bedeutung deutlich herausstellen.

4. Der Aufwand für ein Lehramtsportfolio ist groß; deshalb ist es wichtig, dass auch Sorge getragen wird, dass Auszubildende es hinreichend für ihre Professionalisierungsförderung nutzen. Es scheint deshalb unbedingt geboten, ein solches Portfolio nicht unverbindlich als wünschenswertes Addendum in den Studiengängen für das Lehramt zu nutzen, sondern es fest in Lehre und Prüfungsvorgänge zu integrieren. Dies bedeutet, dass die föderalen Kultusministerien ein solches Portfolio stützen und für politisch wünschenswert halten müssten, damit es in die Prüfungsvorgänge beim Ersten und Zweiten Staatsexamen sinnvoll eingegliedert werden kann. Es scheint absehbar, dass mit Einbezug von Teilen des Portfolios, etwa dem Dossier oder auch dem Reflexionsteil, sich die Abschlussprüfungen werden ändern müssen. Vorstellbar ist hier eine mündliche Abschlussprüfung, in der auf das Lehramtsportfolio explizit und individuell Bezug genommen wird.

5. Reflexion ist ein Kernkonzept und damit der Dreh- und Angelpunkt eines phasenübergreifenden Portfolios. Auch wenn es sich hierbei inzwischen um einen Begriff handelt, der schlagwortartig die unterschiedlichsten Konnotationen hervorruft, ist in den obigen Ausführun- 
gen deutlich geworden, dass die Reflexion von Standards, Kompetenzen, eigenen Leistungen, Wissen und Erfahrungen erst diese ganz unterschiedlichen Aspekte in den Köpfen der Auszubildenden zusammenbringt. Eine regelmäßige, strukturierte, individuelle Beschäftigung mit der eigenen Performanz hilft ihnen dabei, bereits Erreichtes anzuerkennen und sich neue Ziele in der Ausbildung zu setzen. Dies entspricht nicht nur einem kognitiven Lernmodell, sondern auch dem Konzept des selbstständigen Lernens.

6. Der Nutzen eines phasenübergreifenden Portfolios ergibt sich aus den vorangegangenen Punkten. Es kann zunächst als Reflexionsinstrument dienen. Seine Funktionen als Evaluationsinstrument - etwa nach den praktischen Ausbildungsphasen oder in mündlichen Prüfungen ist bereits beschrieben worden. Deutlich geworden ist aber auch seine Dokumentationsfunktion, die es Ausbildenden nach dem Wechsel von einer Phase der Ausbildung in die nächste ermöglicht, den/die Portfolioeigner/-eignerin einzuschätzen. Mentoren/Mentorinnen, Ausbildende im Referendariat, Schulleiter/Schulleiterinnen können einen Einblick in den „öffentlichen“ Teil des Portfolios des angehenden Lehrers/der angehenden Lehrerin erhalten. Bisher keine Beachtung gefunden hat das Portfolio als Dokumentationsinstrument in der Nachwuchsausbildung der Hochschulen, die Doktoranden/Doktorandinnen im Bereich der Fachdidaktiken besser einschätzen können, wenn diese ein Portfolio vorlegen können.

7. Ausgehend von dem Bereich der Fremdsprachenlehrerausbildung, die in Europa aus mehreren Gründen besonders vorangetrieben wurde, ist das wohl im Moment erfolgreichste Modell eines Lehramtsportfolios, das EPOSTL, einer genaueren Betrachtung unterzogen worden. Dabei hat sich gezeigt, dass dieses europäische Rahmenmodell für eine Lehrerausbildung für den deutschen Lehr-/Lernkontext wenig zufriedenstellend ist, da diese Ausbildung immer zwei oder drei Fachwissenschaften und -didaktiken sowie die Grundwissenschaften umfasst. Ein Portfoliomodell für eine deutsche Lehramtsausbildung könnte einige Kompetenzfelder des EPOSTL übernehmen, müsste aber selbst in Hinsicht auf eine fachdidaktische Ausbildung in Sprachen erheblich erweitert werden. Vorzuziehen wäre ein Portfolio, das einen breiteren, auf alle Lehramtsfächer und -didaktiken bezogenen Ansatz vertritt und gleichzeitig eine professionelle Lehrerpersönlichkeit entwickeln möchte. 


\section{Literatur}

Blömeke, Sigrid (2013): Einleitung: Professionelle Kompetenzen im Studienverlauf. In: Blömeke, Sigrid; Bremerich-Vos, Albert; Kaiser, Gabriele; Nold, Günter; Haudeck, Helga; Keßler, Jörg-U.; Schwippert, Knut (Hrsg.):

Professionelle Kompetenzen im Studienverlauf: Weitere Ergebnisse zu Deutsch-, Englisch-, und Mathematiklehrerausbildung aus TEDS-LT. Münster, New York: Waxmann, 7-24.

Blömeke, Sigrid; Bremerich-Vos, Albert; Kaiser, Gabriele; Nold, Günter; Haudeck, Helga; Keßler, Jörg-U.; Schwippert, Knut (2013) (Hrsg.): Professionelle Kompetenzen im Studienverlauf: Weitere Ergebnisse zu Deutsch-, Englisch-, und Mathematiklehrerausbildung aus TEDS-LT. Münster, New York: Waxmann.

Burwitz-Melzer, Eva (2004): Ein Lehramtsportfolio für Fremdsprachenlehrkräfte (LAPF): Auf dem Weg zum ,reflective practitioner'. In: ZFF 15/1, 145-157.

Burwitz-Melzer, Eva (2018): Ein phasenübergreifendes Portfolio in der Lehramtsausbildung. In: Burwitz-Melzer, Eva; Riemer, Claudia; Schmelter, Lars (Hrsg.): Rolle und Professionalität von Fremdsprachenlehrpersonen. Arbeitspapiere der 38. Frübjahrskonferenz. zur Erforschung des Fremdsprachenunterrichts. Tübingen: Narr, 21-31.

Campbell, Dorothy; Cignetti, Pamela Bondi; Melenyzer, Beverly; Nettles, Diane; Wyman, Richard (2001): How to Develop a Professional Portfolio: A Manual for Teachers. Boston: Allyn \& Bacon.

Council of Europe (1997): European Language Portfolio: Proposals for Development. Strasbourg: Council for Cultural Co-Operation.

Council of Europe (2001): Modern Languages: Learning, Teaching, Assessment: A Common European Framework for Reference. Strasbourg: Council of Europe and Cambridge UP.

Grenfell, Mike (2012): The EPOSTL and the European Profile for Language Teacher Education. In: Newby, David (Hrsg.): Insights into the European Portfolio for Student Teachers of Languages. Newcastle upon Tyne: Cambridge Scholars Publishing, 155-174.

Hattie, John (2013): Visible Learning for Teachers. Maximizing Impact on Learning. London: Routledge.

Kelly, Michael; Grenfell Michael (2004): European Profile for Language Teacher Education - A Frame of Reference. http://www.lang.soton.ac.uk/profile/ report/MainReport.pdf [01.03.2019].

Koch-Priewe, Barbara (2013): Das Portfolio in der LehrerInnenbildung Verbreitung, Zielsetzungen, Empirie, theoretische Fundierungen. In: KochPriewe, Barbara; Leonhard, Tobias; Pineker, Anna; Störtländer, Jan Christoph (Hrsg.): Portfolio in der LehrerInnenbildung: Konzepte und empirische Befunde. Bad Heilbrunn: Klinkhardt, 41-73. 
Kohonen, Vilja (2012): Developing autonomy through ELP-oriented pedagogy:

Exploring the interplay of shallow and deep structures in a major change within language education. In: Kühn, Bärbel; Pérez Cavana; Maria Luisa (Hrsg.): Perspectives from the European Language Portfolio: Learner Autonomy and SelfAssessment. London, New York: Routledge, 22-42.

Korthagen, Fred (2004): In search of the essence of a good teacher: towards a more holistic approach in teacher education. In: Teaching and Teacher Education 20, 77-97.

Kupetz, Rita; Ruhm, Hannah (2012): The EPOSTL as a tool to document and reflect on teaching experience abroad. In: Newby, David (Hrsg.): Insights into the European Portfolio for Student Teachers of Languages. Newcastle upon Tyne: Cambridge Scholars Publishing, 217-228.

Lyons, Nora (Hrsg.) (1998): With Portfolio in Hand: Validating the New Teacher Professionalism. New York, London: Teachers College Press.

Neß, Harry (2013): Phasenübergreifendes Professionalisierungs-Portfolio unter Einbeziehung der Validierung des informellen und nicht formalen Lernens. In: Koch-Priewe, Barbara; Leonhard, Tobias; Pineker, Anna; Störtländer, Jan Christoph (Hrsg.): Porffolio in der LehrerInnenbildung: Konzepte und empirische Befunde. Bad Heilbrunn: Klinkhardt, 168-179.

Newby, David; Allan, Rebecca; Fenner, Anna-Brit; Jones, Barry; Komorowska, Hanna; Soghikvan, Kristine (2010): European Portfolio for Student Teachers of Languages A Reflection Tool for Language Teacher Education. European Centre for Modern Languages. http://archive.ecml.at/mtp2/fte/pdf/STPExtract.pdf [01.03.2019].

Newby, David (2012): Introduction. In: Newby, David (Hrsg.): Insights into the European Porffolio for Student Teachers of Languages. Newcastle upon Tyne: Cambridge Scholars Publishing, 1-8.

Rebel, Karlheinz; Wilson, Sybil (2002): Das Professionelle Portfolio in der angelsächsischen Lehrerbildung aus deutscher und kanadischer Sicht. In: Forum Lebrerbildung 36, 60-77.

Schoen, Donald A. (1983): The Reflective Practitioner: How Professionals Think in Action. London: Basic Books.

Ständige Konferenz der Kultusminister der Länder der Bundesrepublik Deutschland (KMK) (2004): Standards für die Lebrerbildung: Bildungswissenschaften. https://www.kmk.org/fileadmin/veroeffentlichungen_beschluesse /2004/2004_12_16-Standards-Lehrerbildung.pdf [01.03.2019]. 
Ständige Konferenz der Kultusminister der Länder der Bundesrepublik

Deutschland (KMK) (2008): Ländergemeinsame inhaltliche Anforderungen für die

Fachwissenschaften und Fachdidaktiken in der Lehrerbildung. https://www.kmk.

org/fileadmin/Dateien/veroeffentlichungen_beschluesse/2008/2008_10_16-

Fachprofile-Lehrerbildung.pdf [01.03.2019].

Ständige Konferenz der Kultusminister der Länder der Bundesrepublik

Deutschland (KMK) (2015): Lehrerbildung für eine Schule der Vielfalt.

https://www.kmk.org/fileadmin/Dateien/veroeffentlichungen_beschluesse/2

015/2015_03_12-Schule-der-Vielfalt.pdf [01.03.2019].

Weinert, Franz E. (Hrsg.) (2001): Leistungsmessungen in Schulen. Weinheim, Basel:

Beltz.

Winter, Felix (2013): Das Portfolio in der Hochschulbildung - Reformimpulse für

Didaktik und Prüfungswesen. In: Koch-Priewe, Barbara; Leonhard, Tobias;

Pineker, Anna; Störtländer, Jan Christoph (Hrsg.): Portfolio in der

LehrerInnenbildung: Konzepte und empirische Befunde. Bad Heilbrunn: Klinkhardt, 15-40. 